\title{
Observation of universal bound state spectrum near a magnetic quantum critical point
}

Quantum Criticality in an Ising Chain: Experimental Evidence for Emergent E8 Symmetry Authors: R. Coldea, D. A. Tennant, E. M. Wheeler, E. Wawrzynska, D. Prabhakaran, M. Telling, K. Habicht, P. Smeibidl, K. Kiefer , Science 327, 177 (2010)

\section{Recommended with a commentary by Ashvin Vishwanath, UC Berkeley}

The quantum Ising chain is one of the simplest quantum many body system, which nevertheless displays highly nontrivial behavior. It is now a staple topic in modern courses on many body theory. The experimental work reviewed here studies a system that is extremely well described by this model. A key prediction regarding the hidden symmetry of this model, made more than two decades back in a landmark theory paper [1], is verified for the first time.

The quantum Ising chain Hamiltonian :

$$
H=-J \sum_{i} \sigma_{i}^{z} \sigma_{i+1}^{z}-h_{x} \sum_{i} \sigma_{i}^{x}
$$

encodes the competition between ferromagnetic exchange that aligns spins along the $\pm z$ direction, leading to spontaneously symmetry breaking, and a transverse magnetic field that aligns spins along the $x$ direction. A quantum phase transition occurs at $h_{x}=J$, between these two phases. Although both phases are gapped, the excitations change character across the transition. In the ferromagnetic phase $\left(h_{x} / J<1\right)$, the lowest energy excitation is a domain wall - a single spin flip corresponds to a pair of domain walls as shown in Figure 1A. Since separating this pair only costs a finite energy, the spin flip, normally the basic excitation in a magnetic system, is said to have fractionalized into a pair of domain walls. Note, the mechanism here is special to one dimension - coupling along other directions leads to a confinement of domain walls. However, in the paramagnetic phase $h_{x} / J>1$, a spin flip like excitation is always the first excited state. Finally, the quantum phase transition itself is well understood theoretically, via a mapping to free fermions, and displays many unique properties.

All of this physics is beautifully confirmed in the experimental paper by R. Coldea et al. 


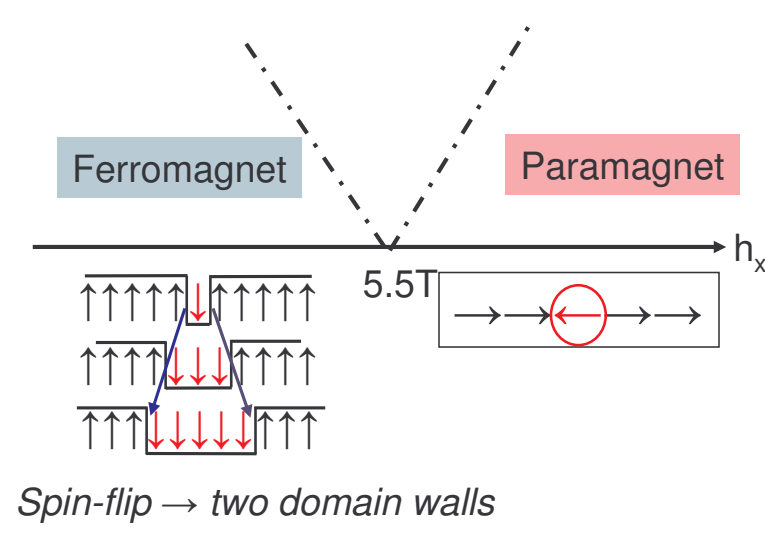

Figure 1A

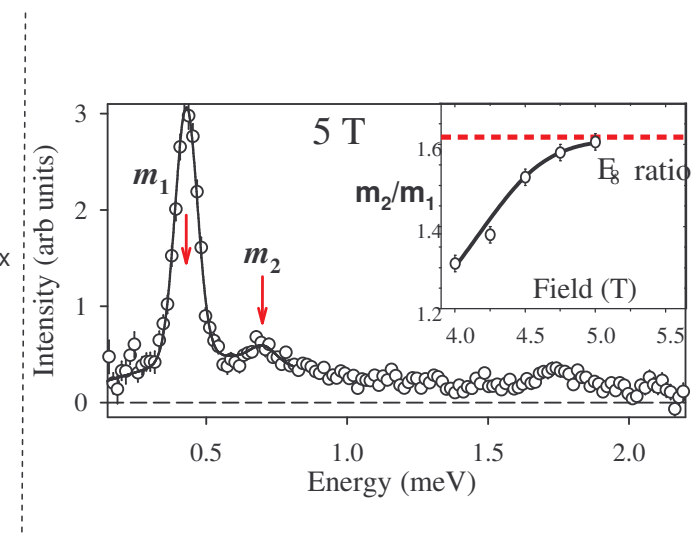

Figure 1B

FIG. 1: (A) Quantum phase transition as a function of transverse magnetic field. On the ferromagnetic side, a single spin flip decays into a pair of domain walls, which are the elementary excitations. (B) Lowest energy bound states on applying a longitudinal field. (inset) The ratio of bound state energies as the critical point is approached is close to the golden mean. [Data from the featured reference]

The first achievement is identifying a suitable material in which this physics can be studied. Such a material must be quasi-one dimensional, with Ising like spins, whose coupling should not be too large, to allow for significant competition from an applied magnetic field. In $\mathrm{CoNb}_{2} \mathrm{O}_{6}$, (a member of the Columbite family of minerals) the $\mathrm{Co}^{2+}$ ions occur in a distorted octahedral environment leading to Ising like spins. The ions are arranged in chains with $90^{\circ}$ Co-O-Co bonds, leading to weak ferromagnetic exchange $(J \approx 20$ Kelvin). Interchain couplings are much weaker and can be neglected to first approximation. Neutron scattering 
experiments on crystals of Co-Columbite then reveal a quantum phase transition out of a magnetically ordered phase, when the transverse magnetic field exceeds 5.5Tesla. The excitation gap closes as this critical field is approached, but the character of excitations is very different on either side. A scattering continuum is observed in the low field ordered phase, corresponding to the fact that a single spin flip can decay into a pair of domain walls (spinons). On the high field side though, a sharp excitation is observed.

At low temperatures, the inter-chain couplings begin to matter, and pairs of domain walls now experience a confining potential that grows linearly with separation. This is similar to the confinement of quarks into mesons. The scattering continuum now breaks up into a sequence of bound states, whose energies are calculated within a simple linear well approximation and found to agree well with the data.

Finally, domain walls can also be confined by applying the magnetic field along the Ising axis: $H_{1}=-h_{z} \sum_{i} \sigma_{i}^{z}$. This naturally washes out the transition given there is now no symmetry distinction between the two phases. However, for weak longitudinal fields in the vicinity of critical point, a remarkable theoretical work from the 80's [1] predicts that a hidden symmetry characterized by the Lie group $\mathrm{E}_{8}$ will emerge. The $\mathrm{E}_{8}$ group with 248 generators is the largest of the exceptional Lie group, and continues to intrigue mathematicians. A specific prediction related to this giant symmetry is that a sequence of eight bound states, with energy ratios determined solely by group properties, must occur. In particular, the lowest two bound states (with energy gaps $m_{1}$ and $m_{2}$ ) appear below the two particle continuum continuum $\left(m_{1}<m_{2}<2 m_{1}\right)$, and have a ratio of energies which is just the golden mean $m_{2} / m_{1}=(1+\sqrt{5}) / 2=1.61803 \ldots$ Interestingly, one can either view the $m_{2}$ particle as a bound state of two $m_{1}$ particles, or the $m_{1}$ particle as a bound state of two $m_{2}$ particles. This leads to the golden mean ratio [1]. As shown in Figure 1B, this pair of bound states is clearly observed, and the energy ratio determined is close to the expected value.

Over the last few decades a number of equally exotic phenomena have been theoretically proposed to exist in two and three dimensional magnetic insulators. In the future perhaps a similarly apt experimental realization of such physics will be found.

[1] A. Zamolodchikov, Int. J. Mod. Phys. A 4, 42354248 (1989). 